\title{
Kaempferol attenuates retinal ganglion cell death by suppressing NLRP1/NLRP3 inflammasomes and caspase-8 via JNK and NF-KB pathways in acute glaucoma
}

\author{
Chaobin $\mathrm{Lin}^{1} \cdot$ Fujin $\mathrm{Wu}^{1} \cdot$ Tongmei Zheng $^{1} \cdot$ Xiuchun $\mathrm{Wang}^{1} \cdot$ Yiwei Chen ${ }^{1} \cdot \mathrm{Xiaomin}_{\mathrm{Wu}^{1}}$
}

Received: 30 June 2018 / Revised: 20 August 2018 / Accepted: 22 November 2018 / Published online: 18 December 2018

(c) The Royal College of Ophthalmologists 2018

\begin{abstract}
Aims or purpose Glaucoma is the leading cause of vision loss and blindness in the world. Elucidating the pathogenesis of glaucoma and developing effective treatment should be the priority. Inflammation and oxidative stress play essential roles in glaucoma pathogeneisis. Kaempferol is a natural flavonol and has anti-inflammatory and anti-oxidative activities. In this study, we explored the potential effects of kaempferol on acute glaucoma.

Methods We established the retinal ischemia-reperfusion (I/R) mice model and administrated kaempferol to I/R mice. We monitored the retina thickness change, retinal ganglion cell (RGC) death, caspase-8 and caspase-3 activation, NLRP1/NLRP3 inflammasomes activation, pro-inflammatory cytokines production, and activations of NF- $\mathrm{KB}$ and MAPKs signaling pathways. Results Kaempferol prevented retina thickness change and RGC death in I/R mice. The activations of caspase-8, caspase-3, and NLRP1/NLRP3 inflammasome activation were inhibited by kaempferol. Kaempferol prevented pro-inflammatory cytokines productions in I/R mice. The activation of NF- $\mathrm{KB}$ and JNK signaling pathways was also inhibited by Kaempferol in I/R mice. Conclusion Kaempferol attenuated retinal ganglion cell death by suppressing NLRP1/NLRP3 inflammasomes and caspase-8 via inhibiting NF- $\mathrm{KB}$ and JNK pathways in acute glaucoma.
\end{abstract}

\section{Introduction}

Glaucoma is the leading cause of worldwide irreversible vision loss and blindness [1]. The damage to the optic nerve and the progressive degeneration of retinal ganglion cells (RGCs) are two major characteristics of glaucoma [2]. Although the pathogenesis of glaucoma is not fully elucidated, high intraocular pressure (IOP), increased oxidative stress, inflammation, and glutamate neurotoxicity have been implicated in glaucoma pathogenesis [3].

Trabecular meshwork blockage allows the build-up of aqueous humor and results in a rapid increase in IOP. Elevated IOP causes retinal ischemic reperfusion (I/R) injury. I/R contributes to cellular damage and causes RGC death glaucoma [4]. Oxidative stress is a key step in the progression of

Xiaomin $\mathrm{Wu}$

qzwuxm@sina.com

1 The First Hospital of Quanzhou Affiliated to Fujian Medical University, No. 250 East Street, Quanzhou 362000 Fujian Province, China glaucoma and is connected to RGC apoptosis [5]. RGC apoptosis is an important component of glaucoma pathophysiology. Apoptosis is a process of programmed cell death requires activities of caspases (cysteine aspartylspecific proteases) family [6]. Caspase- 8 is the initiator caspase which triggers cell apoptosis and Caspase- 3 is the effector caspase in apoptosis [7]. In glaucoma, the NF- $\mathrm{KB}$ signaling pathway is activated and results in the production of pro-inflammatory cytokines, which cause RGC death in retina [8]. Interestingly, NF- $\kappa \mathrm{B}$ inhibitors have been shown to protect neurons against damage in the retina, suggesting that prevention of NF- $\mathrm{KB}$ activation would be a potential candidate to treat retinal denegation.

Inflammasomes are implicated in several $\mathrm{I} / \mathrm{R}$ diseases. In I/R diseases, NOD-like receptor (NLR) families are activated and result in the processing of pro-IL-1 $\beta$ into it mature forms in a caspase-1-dependent manner. NLRP3 inflammasome has been reported to act as a sensor to detect the injury and then amplify the damage signal, leading to subsequent RGC loss [9]. In contrast, inhibiting NLRP3 inflammasome activation protected diabetic retinopathy, suggesting inhibition of NLRP3 inflammasome activation would be another potential candidate to treat retinal denegation [10]. 
Although much effort has been devoted to explore effective therapies to attenuate RGC death, there are still no practically effective drugs [11]. The mechanisms which are related to RGC death and glaucoma pathogenesis could be potential targets of effective therapies [12]. Kaempferol is a natural flavonol found in vegetables and fruits. Kaempferol has been reported to modulate signaling transduction pathways involved in apoptosis, angiogenesis, inflammation, oxidative stress, and metastasis [13]. Kaempferol has been shown to have anti-inflammatory activity targeting the NF$\kappa \mathrm{B}$ pathway [14], suppressing the mitogen-activated protein kinase (MAPK) signaling pathway [15], and NLRP3 inflammasome activation [16]. The anti-oxidative stress activities of kaempferol have also been reported [17]. As $\mathrm{NF}-\mathrm{KB}$ and NLRP3 inflammasome plays essential role in glaucoma, the anti-inflammation activities of kaempferol by targeting NF- $\mathrm{kB}$ and NLRP3 suggested potential effects of kaempferol on glaucoma. Here, we explored the effect of kaempferol on acute glaucoma and reported that kaempferol prevented RGC death and inhibited NLRP1/NLRP3 inflammasome, NF- $\mathrm{KB}$ and JNK2 singling pathways.

\section{Materials and methods}

\section{Retinal ischemia-reperfusion (IR) mice model}

Six- to eight-week C57BL/6 female mice were housed in a 10-14-h light-to-dark-cycle environment. The temperature was kept at $22 \pm 3{ }^{\circ} \mathrm{C}$. Mice were anesthetized by intraperitoneally injecting $4.3 \%$ chloral hydrate with $10 \mathrm{ml} / \mathrm{kg}$ concentration. Pupils were dilated with $1 \%$ tropicamide, and corneas were anesthetized with $0.5 \%$ tetracaine hydrochloride eye drops topically. To increase the intraocular pressure (IOP), the anterior chamber of the right eye was cannulated with a 30-gauge needle and balance salt solution (Tono-Pen XL) was added to maintain at $70 \mathrm{mmHg}$ for $60 \mathrm{~min}$. The left eye was untreated and used as a control. After $60 \mathrm{~min}$, the needle was withdrawn gradually and tobramycin was applied to avoid bacterial infection. Mice were killed at $48 \mathrm{~h}$ after the procedure.

\section{Kaempferol administration}

Kaempferol was purchased from Sigma-Aldrich (St. Louis, MO, USA). After induction of $\mathrm{I} / \mathrm{R}$ injury, mice were randomly divided. Normal mice without treatment of ischemiareperfusion and kaempferol were used as a control group. PBS was used as a vehicle control and I/R mice in vehicle control group were fed with $2 \mu \mathrm{l}$ of PBS. For I/R + kaempferol group, I/R mice were administered intragastrically with kaempferol $100 \mathrm{mg} / \mathrm{kg}$ body weight. Mice were killedand eyes were enucleated $48 \mathrm{~h}$ after administration.

\section{Histological examination}

Histological examination was performed as described previously [18]. Mice eyes were enucleated, and then the whole eyes were fixed with $4 \%$ paraformaldehyde overnight. Next day samples were embedded in paraffin. Four 4-mm-thick sections through the optic nerve of each eye were cut, and sample slices of $10 \mu \mathrm{m}$ per slide were further produced. Every twentieth slice was selected for tissue staining for hematoxylin and eosin (HE) staining. Total retinal thickness (from inner to outer limiting membrane) was measured in four adjacent areas within $1 \mathrm{~mm}$ distance to the optic nerve center using Axiovision Microscopy software (Carl Zeiss MicroImaging Inc., Oberkochen, Germany).

\section{Fluoro-gold (FG) labeling of retinal ganglion cells (RGC)}

FG labeling of RGC was performed as described previously [19]. Briefly, mice were allowed 7 days for retrograde transport of FG before kill to ensure proper RGC labeling. Mice were anesthetized by i.p. injection of a mixture of 100 $\mathrm{mg} / \mathrm{kg}$ ketamine and $10 \mathrm{mg} / \mathrm{kg}$ xylazine and then placed in a stereotactic apparatus (Stoelting). In total, $1 \mu \mathrm{L}$ of $4 \%$ FG (Invitrogen, Carlsbad, CA, USA) diluted in saline (weight/ volume) was injected into both superior colliculi. Fortyeight hours after reperfusion, FG-positive RGCs were quantitated with a fluorescent microscope AxioImager (Carl Zeiss, Germany) after retinal flat mount preparation. Survived RGCs (gold dots) were counted by using Image Pro Plus (Version 6.0; Media Cybernetics).

\section{Western blot}

The whole retina was isolated from the eyeball and then lysed in RIPA buffer to make the homogenates. In some experiment, nuclear proteins were isolated using NE-PER ${ }^{\mathrm{TM}}$ nuclear and cytoplasmic extraction reagents (Thermo Fisher, Waltham, MA, USA). The whole-protein concentrations were measured using Bradford Protein Assay (Bio-Rad, Hercules, CA, USA). Total $25 \mu \mathrm{g}$ protein was loaded on $12 \%$ SDS-PAGE. After transfer, membranes were blocked by $5 \%$ non-fat milk (Bio-Rad, USA) for $1 \mathrm{~h}$ at room temperature and then incubated with different primary antibodies: anti-active Caspase-3 (Abcam, Cambridge, MA, USA), anti-cleaved Caspase-8 (Cell Signaling, Danvers, MA, USA), anti-NALP1 (Cell Signaling, USA), antiNALP3 (Cell Signaling, USA), anti-ASC (Cell Signaling, USA), anti-Capase-1p20 (Santa Cruz, Dallas, TX, USA), anti- $\beta$ actin (Sigma, USA), anti-p-JNK2 (Thermo Fisher, USA), anti-JNK2 (Cell Signaling, USA), anti-p-ERK1/2 (Cell Signaling, USA), anti-ERK1/2 (Millipore, Billerica, MA, USA), anti-p-p38 (Cell Signaling, USA), anti-p38 (Cell 
Signaling, USA), anti-p-IKK $\alpha \beta$ (Sigma, MO, USA), antiIKK $\alpha$ (Abcam, USA), anti-NF- $\mathrm{KB}$ p65 (Thermo Fisher, USA), and anti-lamin B (Abcam, USA). The primary antibodies were incubated at $4{ }^{\circ} \mathrm{C}$ for overnight. Next day, HRPconjugated corresponding secondary antibodies were incubated at room temperature for $1 \mathrm{~h}$. After wash, chemiluminescent substrate (Thermo Fisher Scientific, Waltham, MA) was used to detect the bands. GS- $900^{\mathrm{TM}}$ Calibrated Densitometer and Image Lab (Bio-Rad, Hercules, CA, USA) were used for quantitation following manufacturer's instructions.

\section{Quantitative real-time PCR (RT-PCR)}

RNeasy mini kit (Qiagen, Valencia, CA, USA) was used to isolate total RNA of the retina. The subsequent cDNA was synthesized by using the Quatitect reverse transcription kit (Qiagen, USA). Real-time PCR was performed using SYBR Green Master Mix (Qiagen, USA) following manufacturer's instructions. Samples were normalized to internal control $\beta$-actin. Primer sequences used for RT-PCR were as follows: tumor necrosis factor $\alpha$ (TNF- $\alpha)$ forward primer: 5'-CGTGGAACTGGCAGAAGAGG-3' and reverse primer: 5'-CTGCCACAAGCAGGAATGAG-3', iNOS forward primer: 5'-ACAACAGGAACCTACCAGCTCA-3' and reverse primer: 5'-GATGTTGTAGCGCCTGTGTGTCA-3', interleukin 6 (IL-6) forward primer: 5'-CCAGAAA CCGCTATGAAGTTCC-3' and reverse primer: 5'-GTT GGGAGTGGTATCCTCTGTGA-3', interleukin $1 \beta$ (IL-1 $\beta$ ) forward primer: 5'-GTTCCCATTAGACAACTGCAC TACAG-3' and reverse primer: 5'-GTCGTTGCTTGGTTCT CCTTGTA-3', cyclooxygenase 2 (COX-2) forward primer: $5^{\prime}$-CCAGATGATATCTTTGGGGAGAC- $3^{\prime}$ and reverse primer: 5'-CTTGCATTGATGGTGGCTG-3', $\beta$-actin forward primer: 5'-GGCGGACTATGACTTAGTTG-3' and reverse primer: 5'-AAACAACAA TGTGCAATCAA-3'.

\section{Statistical analysis}

All data were expressed as mean $\pm \mathrm{SD}$ and one-way ANOVA was used for difference analysis. The difference was considered as significant when $p<0.05$.

\section{Results}

\section{Kaempferol inhibited IOP-induced retinal thickness change and prevented RGC death}

The retina layers contain ganglion cell layer (GCL), inner nuclear layer (INL), inner plexiform layer (IPL), outernuclear layer (ONL), and outer plexiform layer (OPL). First, we analyzed the thickness change in control, I/R and I/R + kaempferol groups of mice. As shown in Fig. 1a, I/R mice had decreased retinal thickness when compared with normal mice, indicating elevated IOP caused retinal thickness change. In contrast, administration of kaempferol prevented the decreasing of retinal thickness in $I / R$ mice and the retinal thickness of $I / R+$ kaempferol mice was significantly higher than that of $\mathrm{I} / \mathrm{R}$ mice, indicating kaempferol ameliorated the IOP-induced pathology. The retinal thickness was correlated to RGC death [20]. We further analyzed the RGC death among these three groups of mice. As shown in Fig. 1b, using FG labeling, we found there was obvious less RGC cells in I/R mice than in normal mice, indicating IOPinduced cell death in mice. Once $I / R$ mice were treated with kaempferol, there was no obvious cell loss in $I / R$ mice. After quantitation, kaempferol-treated I/R mice had significantly higher $R G C$ survival rate than that $I / R$ mice. Thus, our data suggested that kaempferol prevented IOPinduced cell death.

\section{Kaempferol prevented caspase- 3 and caspase- 8 activation}

Elevated IOP induced RGC apoptosis through activating caspase-3 and caspase-8 [21]. As kaempferol inhibited RGC death, we continued to determine the effects of kaempferol on caspase- 3 and caspase- 8 activation by western blot. Consistent to previous reports, we found there was obvious increased active caspase-3 (Fig. 2a) and active caspase- 8 (Fig. 2b) in I/R mice. Kaempferol significantly decreased protein levels of both active caspase- 3 and caspase- 8 , indicating inhibitions of caspase- 3 and caspasae- 8 activation.

\section{Kaempferol prevented NLRP1/ NLRP3 inflammation activation}

NLRP1/NLRP3 inflammasome activation was implicated in glaucoma [19]. IOP induced the elevated expression of inflammasome components NLRP1 (Fig. 3a), NLRP3 (Fig. 3b), ASC (Fig. 3c), and active caspase-1 (Fig. 3d). Administration of kaempferol significantly decreased the levels of all four proteins, indicating kaempferol prevented NLRP1/NLRP3 inflammasome activation.

\section{Kaempferol inhibited inflammatory cytokines production and oxidative stress}

Both inflammatory cytokines and oxidative stress played important roles in the process of glaucoma [22, 23]. We analyzed the effects of kaempferol on inflammatory cytokines production and oxidative markers in $\mathrm{I} / \mathrm{R}$ mice retina by RT-PCR. We found that there were increased mRNA levels of interleukin $1 \beta$ (IL-1 $\beta$ ) (Fig. 4a), tumor necrosis factor $\alpha$ (TNF- $\alpha$ ) (Fig. 4b), interleukin 6 (IL-6) (Fig. 4c), 

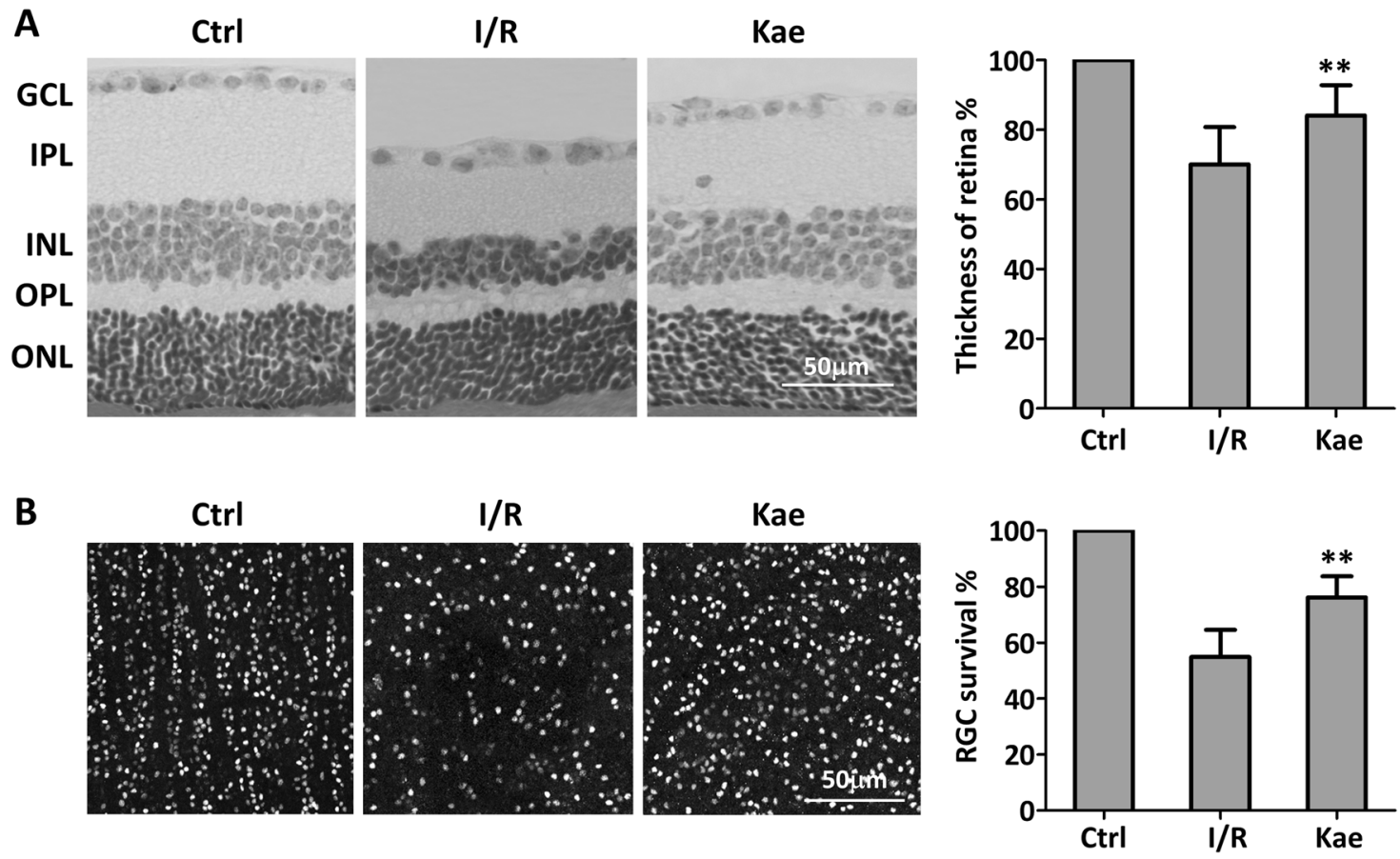

Fig. 1 The effect of kaempferol on IOP-induced RGC death. a Hematoxylin and eosin staining of retinal cross-sections showed difference of RGCs and the mean of the retinal tissue thickness among different groups $48 \mathrm{~h}$ after reperfusion. GCL ganglion cell layer, INL inner nuclear layer, IPL inner plexiform layer, ONL outernuclear layer,
OPL outer plexiform layer. b FG labeling showed that the number of surviving cells in the RGC layer after IOP-induced injury compared with controls. $N=5$ mice. Data are shown as mean \pm SD. $* * P<0.01$ vs. Ctrl group (one-way ANOVA analysis)
Fig. 2 The effect of kaempferol on caspase- 8 and caspase- 3 activations in the retina of IOPinduced mice. a Western blot detected expression of active caspase- 8 protein. b Western blot detected expression of active caspase- 3 protein. $N=5$ mice. Data are shown as mean \pm SD. $* * P<0.01$ vs Ctrl group (one-way ANOVA analysis)
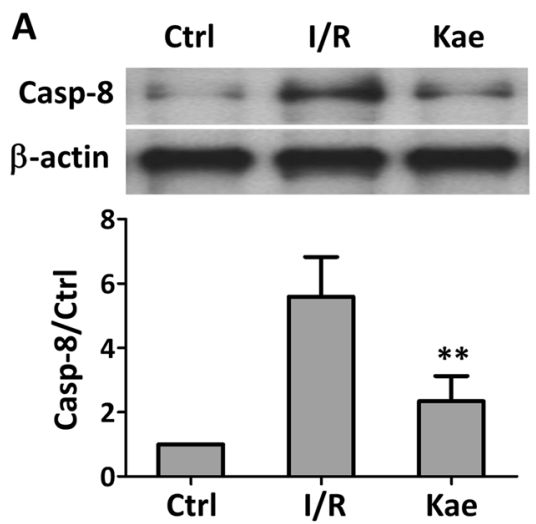
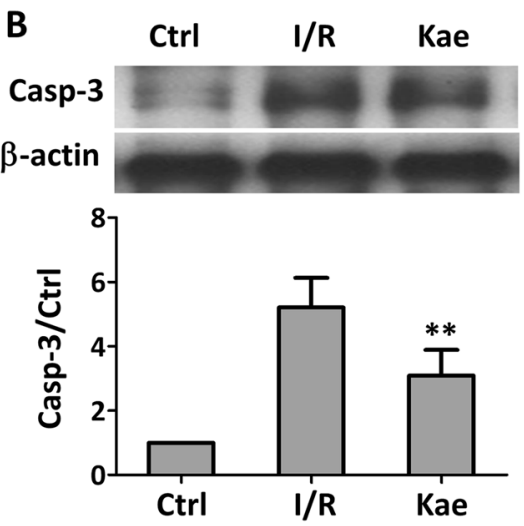

inducible nitric oxide synthase (iNOS) (Fig. 4d), and cyclooxygenase 2 (COX-2) in I/R mice retina (Fig. 4e). In contrast, kaempferol significantly decreased the mRNA levels of all five proteins, indicating kaempferol not only inhibited inflammatory cytokines production but also attenuated oxidative stress.

\section{Kaempferol inhibited activations of NF-KB and JNK signaling pathways}

Both NF- $\mathrm{KB}$ and MAPK signaling pathways were responsible for inflammation response and implicated in glaucoma [24]. We further explored the effects of kaempferol on
NF- $\kappa B$ and MAPK signaling pathway activations. In I/R mice, the MAPK signaling pathways were activated as we detected increased levels of phosphorylated-JNK2 (p-JNK2) (Fig. 5a), phosphorylated ERK1/2 (p-ERK1/2) (Fig. 5b), phosphorylated-p38 (p-p38) (Fig. 5c). Administration of kaempferol in $\mathrm{I} / \mathrm{R}$ mice significantly decreased levels of $\mathrm{p}$-JNK2 but not $\mathrm{p}$-ERK1/2, p-p38, indicating that kaempferol inhibited IOP induced activation of the JNK2 signaling pathway. Similarly, we detected increased level of phosphorylated IKK $\alpha / \beta$ (p-IKK $\alpha / \beta)$ (Fig. $5 \mathrm{~d})$ and nuclear NF- $\mathrm{KB}$ p65 (Fig. 5e) in the retina of $\mathrm{I} / \mathrm{R}$ mice, indicating IOP also induced $\mathrm{NF}-\kappa \mathrm{B}$ activation. The kaempferol treatment significantly decreased levels of both 
A

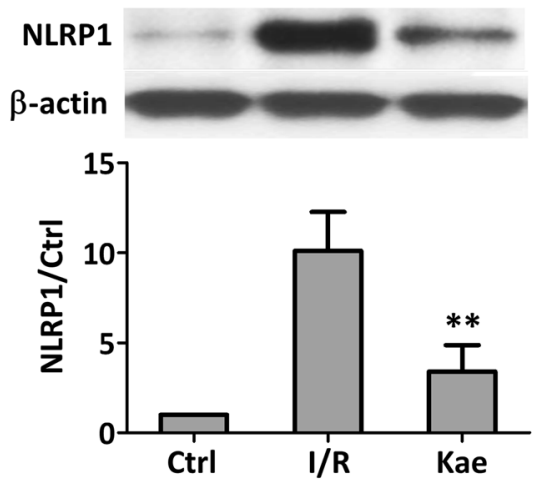

C
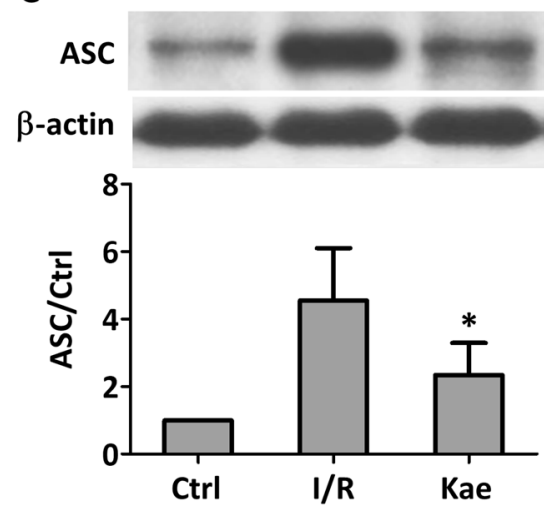

Fig. 3 The effect of kaempferol on the activation of NLRP3 inflammasome in the retina of IOP-induced mice. a-d Western blot detected expression of NLRP1, NLRP3, ASC and caspase-1. $N=5$ mice.
B

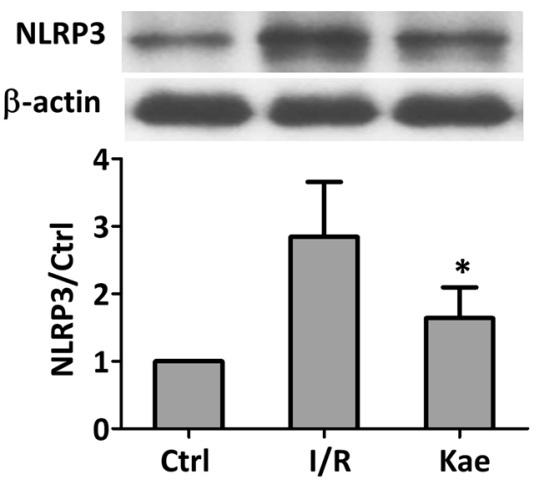

D

Casp-1

\section{$\beta$-actin}

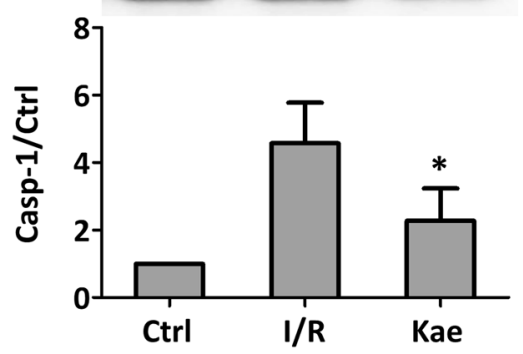

Data are shown as mean $\pm \mathrm{SD}$. $* P<0.05, * * P<0.01$ vs. Ctrl group (one-way ANOVA analysis)
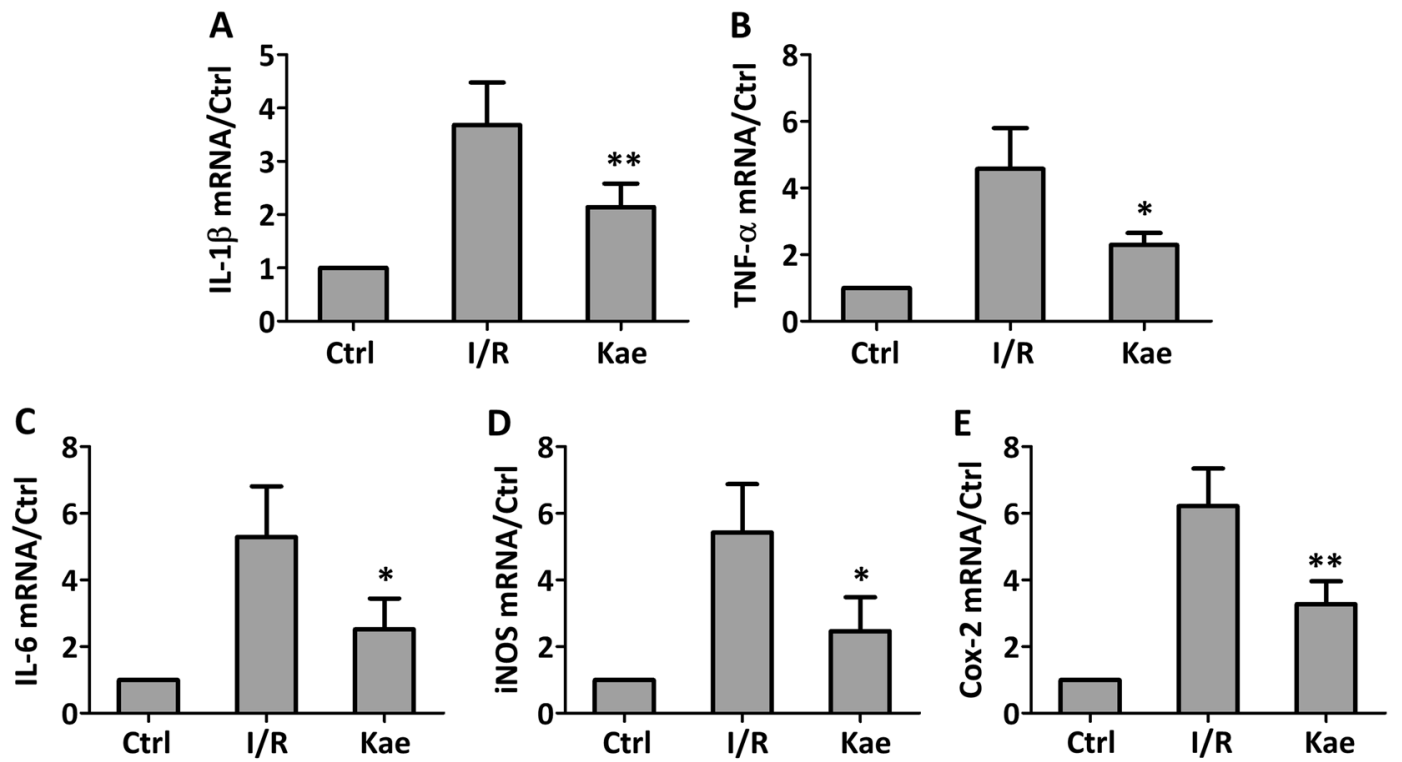

Fig. 4 The effect of kaempferol on the productions of proinflammatory cytokines in the retina of IOP-induced mice. a-e qPCR detected expression of IL-1 $\beta$, TNF- $\alpha$, IL-6, iNOS, and Cox-2.

$N=5$ mice. Data are shown as mean $\pm \mathrm{SD} . * P<0.05, * * P<0.01$ vs. Ctrl group (one-way ANOVA analysis) 
A

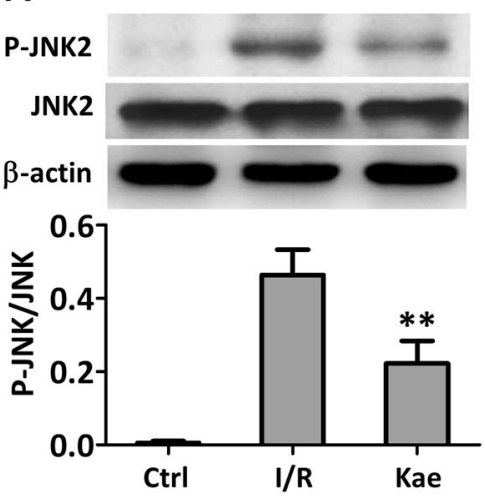

D
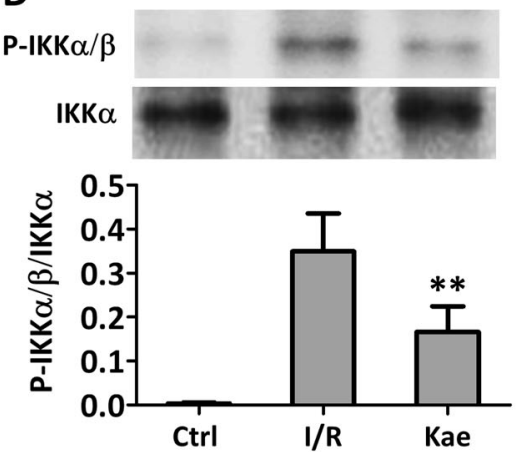

Fig. 5 The effect of kaempferol on the MAPKs and NF- $\mathrm{BB}$ activation in the retina of IOP-induced mice. a-e Western blot detected expression of p-JNK, p-ERK, p-p38, p-IKKs, and p65. $N=5$ mice. Data are

$\mathrm{p}-\mathrm{IKK} \alpha / \beta$ and nuclear NF- $\kappa \mathrm{B}$ p65, indicating kaempferol inhibited IOP induced activation of NF- $\mathrm{KB}$.

\section{Discussion}

Glaucoma is a family of neurodegenerative diseases characterized by the loss of retinal ganglion cells (RGC), which ultimately leads to visual loss. Thus, developing treatments to prevent RGC death and preserve RGC in glaucoma should be the priority [12].

Several rodent models were established for glaucoma studies. As elevated intraocular pressure (IOP) is strongly associated with the progression of glaucoma, rodent models of glaucoma that involve optic nerve damage mediated through ocular hypertension are most commonly used [25]. Retinal ischemia-reperfusion (I/R) is a pathophysiological process contributing to cellular damage in glaucoma, and rodent models of I/R injury are providing significant insights into mechanism and treatment strategies for human I/R injury [26]. Upon I/R mice model, we found there was RGC loss 48 $\mathrm{h}$ after $\mathrm{I} / \mathrm{R}$. I/R mice model is a common used model to explore RGC survival and underlying mechanism, and investigate potential treatment to prevent glaucoma. In current
C
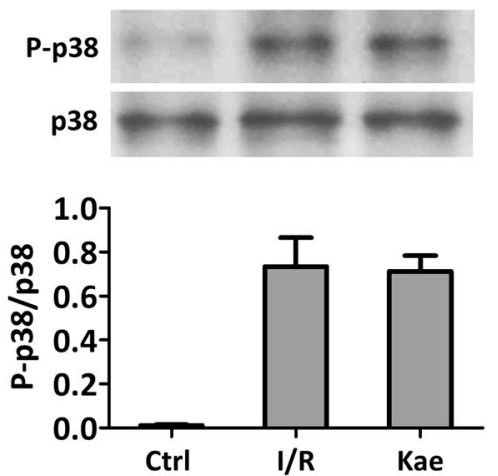

\section{E}

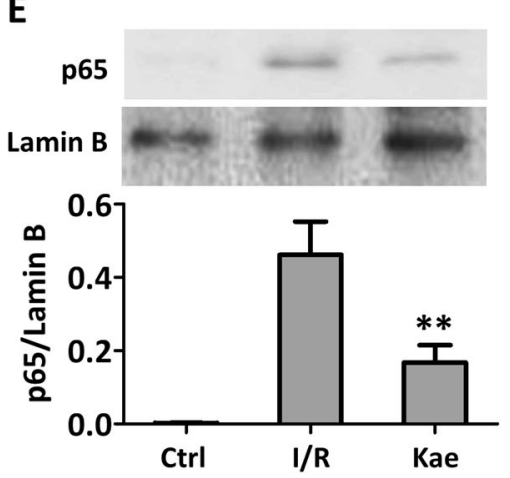

shown as mean $\pm \mathrm{SD}$. $* P<0.05, * * P<0.01$ vs. Ctrl group (one-way ANOVA analysis)

study, we identified that kaempferol prevented I/R-induced retina thickness change by inhibiting RGC death. In addition, we further elucidated that kaempferol inhibited activation of caspase-8, capase-3 and NLRP1/NLRP3 inflammasome, prevented I/R-induced inflammatory cytokine production and oxidative stress by suppressing JNK and NF- $\kappa B$ pathways. Our data strongly suggested that kaempferol could be used as a potential treatment for glaucoma.

The pathophysiology of glaucoma is not well understood while it is believed that multiple factors contribute to it [27]. The elevated IOP results in RGC apoptosis and the correlation of elevated IOP level and RGC axon loss has been reported [28]. In current study, we found that kaempferol prevented RGC loss by inhibiting the caspase- 8 and caspase-3 activation, indicating that kaempferol inhibited RGC apoptosis. The anti-apoptosis activity of kaempferol has been reported. Ruiz et al. reported that kaempferol inhibited caspase- 3 activation and apoptosis in the vascular smooth muscle [29].

Both inflammatory cytokines and oxidative stress contributed to pathogenesis of glaucoma. With identified antioxidative and anti-inflammation activities in several other diseases models, kaempferol was explored for antioxidative and anti-inflammatory effects in $\mathrm{I} / \mathrm{R}$ mice. 
We found that the pro-inflammatory cytokines, iNOS and COX-2 levels were significantly decreased in retina of kaempferol-treated $\mathrm{I} / \mathrm{R}$ mice, indicating the antiinflammatory and anti-oxidative activities of kaempferol also existed in acute glaucoma model. As oxidative stress caused apoptosis in RGC [30], our data indicated that the anti-oxidative activity of kaempferol contributed to its ability to inhibit RGC apoptosis.

A recent publication described the involvement of inflammasome in acute glaucoma [19]. Using the I/R mice model, Chi et al. reported that in acute glaucoma, TLR4 lead to increased caspase- 8 expression and resulted in increased IL-1 $\beta$ expression and RGC death via the caspase1-dependent pathway, which involved NLRP1/NLRP3 inflammasomes. Inhibition of caspase- 8 activation significantly attenuates RGC death by downregulating the activation of NLRP1 and NLRP3. Our findings demonstrated that I/R-induced NLRP1/NLRP inflammasome and caspase-1 activation while kaempferol treatment prevented the inflammasome and caspase-1 activation and downstream IL-1 $\beta$ production.

The downstream signaling of TLR includes mitogenactivated protein kinases (MAPKs), interferon regulatory

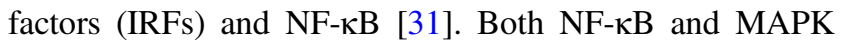
signaling pathways have been implicated in glaucoma $[24$, 32]. Our findings demonstrated that in I/R mice, NF- $\mathrm{KB}$ p65 nuclear translocation and phosphorylation of MAPK JNK2, $\mathrm{p} 38$, and ERK were upregulated, indicating NF- $\mathrm{kB}$ and MAPK signaling pathways were activated during glaucoma. Kaempferol has been reported to regulate both MAPKs and NF-KB signaling pathways [15]. Chen et al. demonstrated that in a LPS-induced acute lung injury mice model, kaempferol attenuated pulmonary edema and significantly reduced LPS-induced pro-inflammatory cytokine production by inhibiting MAPKs and NF- $\mathrm{KB}$ signaling pathways. Our data demonstrated that in $\mathrm{I} / \mathrm{R}$ mice, kaempferol also prevented NF-אB p65 nuclear translocation and JNK but not ERK or p38 phosphorylation, suggesting that kaempferol exerted its anti-glaucoma role via interfering with the NF$\mathrm{\kappa B}$ and JNK pathways.

In conclusion, our current study provided the compelling evidence that kaempferol prevented RGC death by inhibiting the activation of caspase-8, caspase-3, and NLRP1/ NLRP3 inflammasomes, preventing the production of proinflammatory cytokines through inhibiting NF- $\mathrm{KB}$ and JNK pathways.

\section{Summary}

\section{What was known before}

- Glaucoma is the leading cause of vision loss and blindness in the world. Elucidating the pathogenesis of glaucoma and developing effective treatment should be the priority. Inflammation and oxidative stress play essential roles in glaucoma pathogenesis. Kaempferol is a natural flavonol and has anti-inflammatory and antioxidant activities.

\section{What this study adds}

- Kaempferol attenuated retinal ganglion cell death by suppressing NLRP1/NLRP3 inflammasomes and caspase- 8 via inhibiting NF- $\mathrm{KB}$ and JNK pathways in acute glaucoma.

\section{Compliance with ethical standards}

Conflict of interest The authors declare that they have no conflict of interest.

Publisher's note: Springer Nature remains neutral with regard to jurisdictional claims in published maps and institutional affiliations.

\section{References}

1. Resnikoff S, Pascolini D, Etya'ale D, Kocur I, Pararajasegaram R, Pokharel GP, et al. Global data on visual impairment in the year 2002. Bull World Health Organ. 2004;82:844-51.

2. Sacca SC, Gandolfi S, Bagnis A, Manni G, Damonte G, Traverso $\mathrm{CE}$, et al. The outflow pathway: a tissue with morphological and functional unity. J Cell Physiol. 2016;231:1876-93.

3. Kimura A, Namekata K, Guo X, Noro T, Harada C, Harada T. Targeting oxidative stress for treatment of glaucoma and optic neuritis. Oxid Med Cell Longev. 2017;2017:2817252.

4. Weinreb RN, Aung T, Medeiros FA. The pathophysiology and treatment of glaucoma: a review. JAMA. 2014;311:1901-11.

5. Erb C, Heinke M. Oxidative stress in primary open-angle glaucoma. Front Biosci (Elite Ed). 2011;3:1524-33.

6. Farkas RH, Grosskreutz CL. Apoptosis, neuroprotection, and retinal ganglion cell death: an overview. Int Ophthalmol Clin. 2001;41:111-30.

7. Guo L, Moss SE, Alexander RA, Ali RR, Fitzke FW, Cordeiro MF. Retinal ganglion cell apoptosis in glaucoma is related to intraocular pressure and IOP-induced effects on extracellular matrix. Invest Ophthalmol Vis Sci. 2005;46:175-82.

8. Halder SK, Matsunaga H, Ishii KJ, Ueda H. Prothymosin-alpha preconditioning activates TLR4-TRIF signaling to induce protection of ischemic retina. J Neurochem. 2015;135:1161-77.

9. Feng L, Liu X. NLRP3 inflammasome in retinal ganglion cell loss in optic neuropathy. Neural Regen Res. 2016;11:1077-8.

10. Hao J, Zhang H, Yu J, Chen X, Yang L. Methylene blue attenuates diabetic retinopathy by inhibiting NLRP3 inflammasome activation in STZ-induced diabetic rats. Ocul Immunol Inflamm. 2018. https://doi.org/10.1080/09273948.2018.1450516

11. Tian K, Shibata-Germanos S, Pahlitzsch M, Cordeiro MF. Current perspective of neuroprotection and glaucoma. Clin Ophthalmol. 2015;9:2109-18.

12. Xu Y, Yang B, Hu Y, Lu L, Lu X, Wang J, et al. Wogonin prevents TLR4-NF-kappaB-medicated neuro-inflammation and 
improves retinal ganglion cells survival in retina after optic nerve crush. Oncotarget. 2016;7:72503-17.

13. Chen AY, Chen YC. A review of the dietary flavonoid, kaempferol on human health and cancer chemoprevention. Food Chem. 2013;138:2099-107.

14. Kadioglu O, Nass J, Saeed ME, Schuler B, Efferth T. Kaempferol Is an anti-inflammatory compound with activity towards NF-kappaB pathway proteins. Anticancer Res. 2015;35:2645-50.

15. Chen X, Yang X, Liu T, Guan M, Feng X, Dong W, et al. Kaempferol regulates MAPKs and NF-kappaB signaling pathways to attenuate LPS-induced acute lung injury in mice. Int Immunopharmacol. 2012;14:209-16.

16. Lim H, Min DS, Park H, Kim HP. Flavonoids interfere with NLRP3 inflammasome activation. Toxicol Appl Pharmacol. 2018;355:93-102.

17. Samhan-Arias AK, Martin-Romero FJ, Gutierrez-Merino C. Kaempferol blocks oxidative stress in cerebellar granule cells and reveals a key role for reactive oxygen species production at the plasma membrane in the commitment to apoptosis. Free Radic Biol Med. 2004;37:48-61.

18. Chi W, Chen H, Li F, Zhu Y, Yin W, Zhuo Y. HMGB1 promotes the activation of NLRP3 and caspase-8 inflammasomes via NFkappaB pathway in acute glaucoma. J Neuroinflamm. 2015;12:137.

19. Chi W, Li F, Chen H, Wang Y, Zhu Y, Yang X, et al. Caspase-8 promotes NLRP1/NLRP3 inflammasome activation and IL-1beta production in acute glaucoma. Proc Natl Acad Sci USA. 2014;111:11181-6.

20. McKernan DP, Caplis C, Donovan M, O'Brien CJ, Cotter TG. Age-dependent susceptibility of the retinal ganglion cell layer to cell death. Invest Ophthalmol Vis Sci. 2006;47:807-14.

21. Qu J, Wang D, Grosskreutz CL. Mechanisms of retinal ganglion cell injury and defense in glaucoma. Exp Eye Res. 2010;91:48-53.
22. Tong Y, Zhou YL, Zheng Y, Biswal M, Zhao PQ, Wang ZY. Analyzing cytokines as biomarkers to evaluate severity of glaucoma. Int J Ophthalmol. 2017;10:925-30.

23. Izzotti A, Bagnis A, Sacca SC. The role of oxidative stress in glaucoma. Mutat Res. 2006;612:105-14.

24. Zhou X, Li F, Kong L, Tomita H, Li C, Cao W. Involvement of inflammation, degradation, and apoptosis in a mouse model of glaucoma. J Biol Chem. 2005;280:31240-8.

25. Johnson TV, Tomarev SI. Rodent models of glaucoma. Brain Res Bull. 2010;81:349-58.

26. Hartsock MJ, Cho H, Wu L, Chen WJ, Gong J, Duh EJ. A mouse model of retinal ischemia-reperfusion injury through elevation of intraocular pressure. J Vis Exp. 2016;113:e54065.

27. Agarwal R, Gupta SK, Agarwal P, Saxena R, Agrawal SS. Current concepts in the pathophysiology of glaucoma. Indian $\mathrm{J}$ Ophthalmol. 2009;57:257-66.

28. Chauhan BC, Pan J, Archibald ML, LeVatte TL, Kelly ME, Tremblay F. Effect of intraocular pressure on optic disc topography, electroretinography, and axonal loss in a chronic pressureinduced rat model of optic nerve damage. Invest Ophthalmol Vis Sci. 2002;43:2969-76.

29. Ruiz E, Padilla E, Redondo S, Gordillo-Moscoso A, Tejerina T. Kaempferol inhibits apoptosis in vascular smooth muscle induced by a component of oxidized LDL. Eur J Pharmacol. 2006;529:79-83.

30. Maher P, Hanneken A. The molecular basis of oxidative stressinduced cell death in an immortalized retinal ganglion cell line. Invest Ophthalmol Vis Sci. 2005;46:749-57.

31. Kumar H, Kawai T, Akira S. Toll-like receptors and innate immunity. Biochem Biophys Res Commun. 2009;388:621-5.

32. Mammone T, Chidlow G, Casson RJ, Wood JPM. Expression and activation of mitogen-activated protein kinases in the optic nerve head in a rat model of ocular hypertension. Mol Cell Neurosci. 2018;88:270-91. 